\title{
Biogeography-Based Optimization Algorithm for Prolonging Network Lifetime of Heterogeneous Wireless Sensor Networks
}

\author{
Basim Abood ${ }^{1}, \mathrm{Yu} \mathrm{Li}^{1 *}$, Nasseer Bacheche ${ }^{1}$ and Aliaa Hussien ${ }^{2}$ \\ ${ }^{1}$ National Laboratory for Optoelectronics, Huazhong University of Science and \\ Technology Wuhan, China 430074 \\ ${ }^{2}$ Baghdad University, College of Science, Department of astronomy. \\ bas_eng84@ymail.com; *hustlyu@hust.edu.cn;
}

\begin{abstract}
A Wireless Sensor Network (WSN) is a network of distributed sensors that can collect information from a physical environment. Low-cost sensors are the backbone of a WSN. Maximizing the lifetime of sensor networks is the main challenge of a Wireless Sensor Network (WSN), such as the sensor node of the network needs to be prepared with limited battery power. This paper focuses on finding the optimal sink position. Relay nodes have been introduced in conjunction with the sensor nodes to mitigate network geometric deficiencies since in most other approaches the sensor nodes close to the sink become heavily involved in data forwarding and, thus, their batteries are quickly depleted. A Biogeography-Based Optimization (BBO) algorithm is used to locate the optimal sink position with respect to those relay nodes to make the network more energy efficient. The relay nodes communicate with the sink instead of the sensor nodes. Tests show that this approach can save at least $40 \%$ of the energy and prolong the network lifetime.
\end{abstract}

Keywords: Biogeography-Based Optimization, wireless sensor network, sensor node, relay node, lifetime

\section{Introduction}

Sensor nodes are used to provide information by transferring data between themselves [1]. A wireless sensor node is prepared with computing and sensing device, radio transceiver and power components. After the sensor nodes are deployed, they are responsible for self-organizing an appropriate network infrastructure often with multi-hop connections between them.

Then the onboard sensors start collecting information of interest, which is routed towards a sink, and it is assumed that these sensor nodes are aware of the sink location [2].The extension of the network lifetime of Wireless Sensor Networks (WSNs) is an important issue that has not been appropriately solved yet.

Nodes with renewable energy sources (denoted as primary nodes) carry out most message transfer tasks, and nodes armed with conventional chemical batteries (denoted as secondary nodes) are those with fewer communication demands. The key design issue of this network type is the development of a new optimization framework to compute the optimal assignment of renewable energy supplies (primary node assignment) to maximize network lifetime, obtaining the minimum number of energy supplies and their node assignment. A Wireless Sensor Network consists of a large group of autonomous low-cost devices (nodes) distributed over an area that monitor one or several environmental variables of interest. In these networks, the sources are nodes that capture and collaboratively communicate the acquired data (temperature, humidity, lightness, etc.) to the sink node/s, which is/are usually out of their direct coverage. As a consequence, intermediate nodes, in the path between sources and sinks, are in charge of retransmitting the sensed data, selecting, in a distributed manner, the best path to the sink [3]. 
Current node technology and network lifetime have restricted the deployment of WSNs recently. Like many other autonomous electronic devices, the most common source of power for WSN nodes (including the sensing instrumentation and radio communication subsystems) [3]. The distributed schemes offer the opportunity for drastic reductions in communication requirements, at the expense of some performance degradation [4].In a distributed detection system, every sensor node performs some preliminary processing of data and transmits a local decision to the fusion centre. Based on the received data, the fusion centre is responsible for the final decision-making [5].

In this paper, a technique known as Biogeography-Based Optimization (BBO) is used to recover WSN coverage. This method was first proposed in 2008 by Dan Simon [6]. BBO used the migration of species in their habitats to search for a solution to an optimization problem. Similar way to other swarm intelligence algorithms, BBO is simple and robust, which makes it a suitable candidate for solving multi-objective optimization problems such as WSN coverage maximization. The mutation model is employed in BBO to improve the ability of the algorithm to obtain a globally optimum solution. However, this also leads to slow convergence. For WSN nodes with limited energy resources, this can decrease the network lifetime or even reduce the sensor energy.

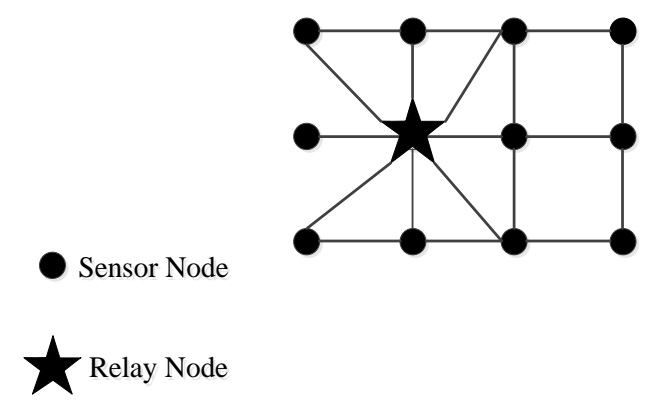

Figure 1. Wireless Sensor Networks Using Relay Nodes

To address these issues, these batteries have two main drawbacks: (i) the nonenvironmental sustainability of their chemical elements, since they can be abandoned in the monitoring area; and (ii) fast depletion when nodes operate intensively. The problem for maximum number of complete cover subsets is difficult, because each subset must achieve complete coverage to the target area, and the WSN can satisfy the surveillance task with only one subset of sensors active at any time. We added this algorithm to improve WSN coverage.

The key motivation of this paper is to optimize the performance of the system with respect to a desired performance criterion, which is specified as the detection error probability at the fusion center. The decision rule at the fusion center, along with the local sensor decision rules, needs to be jointly designed to optimize the specified performance criterion. The goal is to decrease the number of iterations required to find a globally optimum solution for node dispersion. This paper aims to develop a numerical solution for optimal power scheduling in WSNs for correlated observations. The (BBO) algorithm has been proposed to address this issue.

The rest of this paper is organized as follows. Section 2 presents the related work in this paper. Section 3 propose an algorithm of the Biogeography-Based Optimization and includes the chromosome encoding method of, the design of the fitness function, and mutation operations. Experimental results and discussions are presented in Section 4. Finally, Section 5 draws a conclusion for this research. 


\section{Related Work}

WSN lifetime maximization in energy harvesting systems has been tackled in the scientific literature from many different points of view. In a distributed detection system, every sensor node performs some preliminary processing of data and transmits a local decision to the fusion centre. Various methods have been proposed for prolonging the optimal lifetime of a WSN focusing on the device control [7-9], device placement [10], issues of data processing [11], and routing [12-15]. The allocation of different capacity batteries to the network nodes' lifetime maximization is addressed in the work conducted by Long et. al. [16].The Measurement Square Error (MSE) algorithm is proposed by Reise et. al. to maximize the lifetime, characterizing the energy consumption through (MSE) [17]. In other words, maximizing the number of complete cover subsetsis a more direct way of maximizing the network lifetime [18]. Because the point target is the most common phenomenon in practical application, in this paper, the point-coverage problem is considered to maximize the lifetime of a WSN. In [19], a maximum cover using the mixed integer programming (MC-MIP) algorithm is proposed to find the maximum number of complete cover subsets. The first introduced gene algorithm to solve the pointcoverage problem for a WSN by finding the maximum number of complete cover subsets is termed GAMDSC. Recently, a novel hybrid genetic algorithm using a forward encoding scheme (STHGA) was proposed in [20-21].Due to its original definition by [22], the Differential Evolution (DE) algorithm and its variants are perceived as a reliable and versatile population-based heuristic optimization technique [23].

This paper aims to develop a numerical solution for optimal power scheduling in WSNs for correlated observations. The biogeography-based optimization (BBO) algorithm has been proposed to address this issue.

The contribution for this paper is the estimation of the optimum number of nodes, incorporating energy sources, and maximizing network lifetime. Additionally, our logical model is able to acquire the precise location of these nodes in the network regardless of its topology. On the other hand, our research also optimizes the traffic load balancing between a node and its neighbors'. These results will enable a developer or researcher to easily carry out their own deployment/test.

\section{Introduction to Biogeography-Based Optimization}

\subsection{Standard Unconstrained BBO Algorithm}

The Biogeography-Based Optimization (BBO) algorithm, which was developed by Simon [11], was strongly influenced by the equilibrium theory of island biogeography [24]. The basic premise of this theory is that the rate of change in the number of species on an island critically depends on the balance between the immigration of new species onto the island and the emigration of established species. The BBO algorithm operates in a population of individuals called habitats (or islands). Each habitat represents a possible solution to the problem in hand. The fitness of each habitat is determined by its habitat suitability index (HSI), which is a metric that determines the goodness of a candidate solution, and each habitat feature is called a suitability index variable (SIV). Good solutions may have a larger number of species, which represents a habitat with a low HSI, than do poor solutions. The immigration rate $\lambda$ and the emigration rate $\mu$ of each habitat are used to probabilistically share information between habitats. These parameters are affected by the number of species $S$ in a habitat. Maximum immigration rate I occur when the habitat is empty and decreases as more species are added, and maximumemigration rate E occurs when all possible species Smaxare present in the habitat. The immigration and emigration rates when there are $\mathrm{S}$ species in the habitat are given by: 


$$
\begin{aligned}
& \kappa_{s}=I\left(1-\frac{s}{s_{\max }}\right)(1) \\
& \mu_{s}=E\left(\frac{s}{S_{\max }}\right)(2)
\end{aligned}
$$

We have considered a linear migration model where the immigration rate $\lambda_{S}$ and the emigration rate $\mu_{S}$ are linear functions of the number of species $S$ in the habitat, but different mathematical models of biogeography that include more complex variables are presented in [24]. The BBO algorithm can be described with Algorithm 1. The two basic operators that govern the working of $\mathrm{BBO}$ are migration, which is described in Algorithm 2 , and mutation, where rand $(0,1)$ isuniformly distributed random numbers in the interval $[0,1]$. The mutation rate $m(S)$ is inversely proportional to the species count probability $P_{s}$, i.e.,,

$$
m(S)=m_{\max }\left(1-\frac{P_{S}}{P_{\max }}\right)(3)
$$

Where $m_{\max }$ is a user-defined parameter, and $P_{\max }=\max _{S} P_{S}, S=1, \ldots, S_{\max }$. If a habitat is selected for mutation, then a randomly chosen SIV in the habitat is simply replaced with a new randomly generated variable from its range.
Algorithm 1: BBO
1: Initialize a set of solutions (habitats) to a problem.
2: Evaluate the fitness $(\mathrm{k})$ for each solution.
3: Compute $S, \lambda$, and $\mu$ for each solution.
4: Modify habitats (migration) based on $\lambda$ and $\mu$.
5: Mutation based on probability.
6: Implement elitism.
7: Go to step 2 for the next iteration if needed.

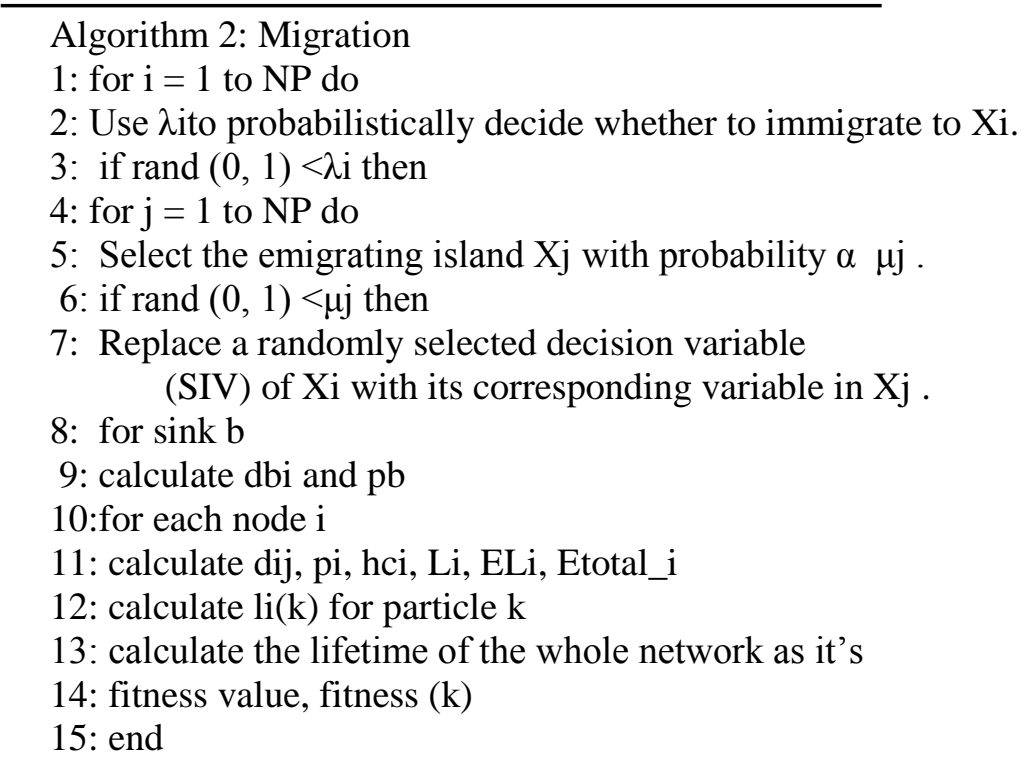

\subsection{Set of Neighbors' of Node i}

It is assumed that the positions of all the nodes are known in terms of $\mathrm{x}$ and $\mathrm{y}$ coordinates. So the distance dij between the nodes $\mathrm{i}$ and $\mathrm{j}$ is calculated according to the following formula:

$$
d_{i j}=\sqrt{\left(x_{i}-x_{j}\right)^{2}+\left(y_{i}-y_{j}\right)^{2}}(4)
$$

Considering dtras the threshold transmission distance for all the nodes, node $\mathrm{j}$ is said to be the neighbor of node $i$ if and only if: 


$$
P_{i}=\left\{j: d_{i j} \leq d_{t r}\right\}(5)
$$

\subsection{Set of Neighbors of the Sink}

As the location of the sink $\mathrm{b}$ is $(\mathrm{xb}, \mathrm{yb})$, therefore the distance from the node $\mathrm{i}$ to the sink $\mathrm{b}$ is:

$$
d_{i j}=\sqrt{\left(x_{b}-x_{i}\right)^{2}+\left(y_{b}-y_{i}\right)^{2}}(6)
$$

Thus we can evaluate the set of neighbors for the sink $b$ by the following equation:

$$
\mathrm{P}_{\mathrm{b}}=\left\{\mathrm{i}: \mathrm{d}_{\mathrm{bi}} \leq \mathrm{d}_{\mathrm{t}}\right\}(7)
$$

\subsection{Calculation of Hop-Count}

The hop count, hc,i , of node $\mathrm{i}$ is calculated using the following formula where hop count distance is expressed as $\mathrm{dt}$ :

$$
h_{c, i}= \begin{cases}{\left[h_{f, i}\right],} & \text { if } h_{f, i} \neq 0 \\ 1, & \text { if } h_{f, i}=0\end{cases}
$$

The hf,iin the above equation can be written as follows where dtis the hop count distance:

$$
\mathrm{h}_{\mathrm{f}, \mathrm{i}}=\frac{\mathrm{d}_{\mathrm{bi}}}{\mathrm{d}_{\mathrm{t}}}
$$

\subsection{Finding the Optimal Link}

In order to find the optimal link for each node to the sink, the concept introduced in Minimum Transmission Energy (MTE) routing [25] has been used, and the link Li for the node iis given by:

$$
\begin{aligned}
& \mathrm{L}_{\mathrm{i}}=\left\{\mathrm{i}, \mathrm{j}: \mathrm{h}_{\mathrm{c}, \mathrm{j}}=\mathrm{n} ; \mathrm{nis}\right. \text { an integer; and } \\
&\left.1 \leq \mathrm{n} \leq \mathrm{h}_{\mathrm{c}, \mathrm{i}}-1\right\}(10)
\end{aligned}
$$

\subsection{Energy Consumption of Each Link}

As in a link, the first node consumes energy on transmission only and other nodes consume energy for both transmission and reception, so energy consumption of each link is:

$$
\mathrm{E}_{\mathrm{L}_{\mathrm{i}}}=\mathrm{h}_{\mathrm{c}, \mathrm{i}} *\left(\mathrm{~h}_{\mathrm{c}, \mathrm{i}}-1\right) * \mathrm{E}_{\mathrm{R}, \mathrm{x}}
$$

\subsection{Energy Consumption of Each Node}

According to Eq. (12), if a node is used to receive ( $n-1)$ times, It should transmit $n$ times. Hence the energy consumption of each node is:

$$
\mathrm{E}_{\mathrm{L}_{\mathrm{i}}}=\mathrm{h}_{\mathrm{c}, \mathrm{i}} *\left(\mathrm{~h}_{\mathrm{c}, \mathrm{i}}-1\right) * \mathrm{E}_{\mathrm{R}, \mathrm{x}}(12)
$$

But if a relay node transmits $\mathrm{n}$ times it also receives $\mathrm{n}$ times. So the energy consumption of each relay node is given by:

$$
\mathrm{E}_{\mathrm{tot} \_1}=\mathrm{n} * \mathrm{E}_{\mathrm{T}, \mathrm{x}}+\mathrm{n} * \mathrm{E}_{\mathrm{R}, \mathrm{x}}
$$

\subsection{Lifetime of node $i$}

The lifetime li (k) of nodei at k-th particle is expressed by:

$\mathrm{l}_{\mathrm{i}(\mathrm{k})}=\frac{\mathrm{E}_{\text {init-i(k)}}}{\mathrm{E}_{\text {tot }-\mathrm{i}(\mathrm{k})}}$

\subsection{Fitness Function}

The fitness function for each particle has been used as: 


$$
\text { fitness }(\mathrm{k})=\underset{\mathrm{i}=1}{\mathrm{Min}}\left\{\mathrm{l}_{\mathrm{i}(\mathrm{k})}\right\}(15)
$$

\section{Simulation of Results}

Once the sensor nodes are deployed, it is often infeasible or undesirable to recharge sensor nodes or replace their batteries. Thus energy conservation becomes crucial for sustaining a sufficiently long network lifetime. So the basic aim behind the design of WSNs is to sustain the network for the required mission. There are normally, in practical systems, certain decisions taken at each layer to improve the network lifetime. In the physical layer, an optimal sink location can greatly improve the sensor network's lifetime.

For a dynamic network this might not be easy or feasible, but for a fixed network the use of a proper design algorithm accurately locating the sink can help save energy. When in active mode, a sensor can carry out its full operations, such as sensing, computation and communication. To maintain those operations, sensors need to consume a relatively large amount of energy. In contrast, a sensor in sleep mode uses only a small amount of energy and can be awoken in a scheduled working interval for full operations. When a subset of sensors in the area can already cover the target area completely, the other sensors can be scheduled to be in sleep mode to save energy; thus, if the number of subsets is maximized, the lifetime of the WSN is prolonged.

We evaluate the performance of our proposed scheme. The required parameters and their values are listed in Table 1. Figure 2 shows the further improvement of the network lifetime as compared to the recent work that was done by Thomas et. al. [26] using the proposed scheme for any cast routing. This Figure depicts that a network lifetime of about $32 \%$ has been increased. The optimal location of the sink is shown in Figure 3 using a circular point. Figure 4 shows the variation of the lifetime with respect to the size of the area over which the nodes are uniformly distributed. From this Figure we can see that if a fixed amount of sensor nodes are uniformly distributed over a larger area, the lifetime of the network decreases. This is because the distance between the nodes increases and thus more energy is spent in transmitting the same amount of data.

Figure 5 and Figure 6 show the changes in the optimal sink location as the size of the area is varied. In Figure 2, we have compared the lifetime when the number of sensor nodes is decreased from 676 to 484 and 196 relay nodes are also used. In the network model it is assumed that in its turn, a sensor node always has some data to send. Moreover, the length of the data is fixed. Now when the number of senor nodes is decreased, at a given period of time the total data sent to the sink is also decreased, which means the data traffic is decreased. In the first case, when only sensor nodes are used without any relay node, the lifetime is measured until the first sensor node is drained of its energy, because when a single sensor node dies, it can damage a complete link to the sink.But in the second case, relay nodes are considered and they are responsible for transferring the data to the sink, and therefore a single sensor node cannot damage a complete data link. So the lifetime and the best position of the sink are located with respect to those relay nodes and thus an improved network lifetime isachieved.

Table 1. Simulation of Parameters

\begin{tabular}{|l|c|}
\hline Parameters & Value \\
\hline Terrain & $1000 \mathrm{~m} \times 1000 \mathrm{~m}$ \\
\hline No. of sensor nodes, $N$ & 676 \\
\hline No. of relay nodes, & 256 \\
\hline Node distribution & Uniform square grid \\
\hline Hop count distance, $d_{t}$ & $61 \mathrm{~m}$ \\
\hline Transmission radius, $d_{t r}$ & $91.5 \mathrm{~m}$ \\
\hline
\end{tabular}




\begin{tabular}{|l|c|}
\hline Interference range, $I$ & $183 \mathrm{~m}$ \\
\hline Propagation model & Free space \\
\hline Initial energy, Einit_I & $2 \mathrm{~J}$ \\
\hline Energy consumed by radio electronics, $E_{\text {elec }}$ & $50 \mathrm{~nJ} / \mathrm{bit}$ \\
\hline Energy consumed by power amplifier, $E_{F s}$ & $10 \mathrm{pJ} \mathrm{bit}-1 \mathrm{~m}^{-2}$ \\
\hline Energy consumed by power amplifier, $E_{T r}$ & $0.0013 \mathrm{pJ} \mathrm{bit}-1 \mathrm{~m}^{-4}$ \\
\hline Data rate & $512 \mathrm{Kbps}$ \\
\hline Data generated by each node & $1000 \mathrm{bit}$ \\
\hline Order of system, $p$ & 2 \\
\hline Particle number, $n$ & 20 \\
\hline Maximum iteration, $m$ & 800 \\
\hline Learning factors, $c 1, c 2$ & 1.5 each \\
\hline Inertia weight, $w$ & $0.1-1.2$ \\
\hline Upper bound & $1000 \mathrm{~m}(\mathrm{x}$ and y axes \\
& both $)$ \\
\hline Lower bound & 0 (x and y axes both) \\
\hline
\end{tabular}

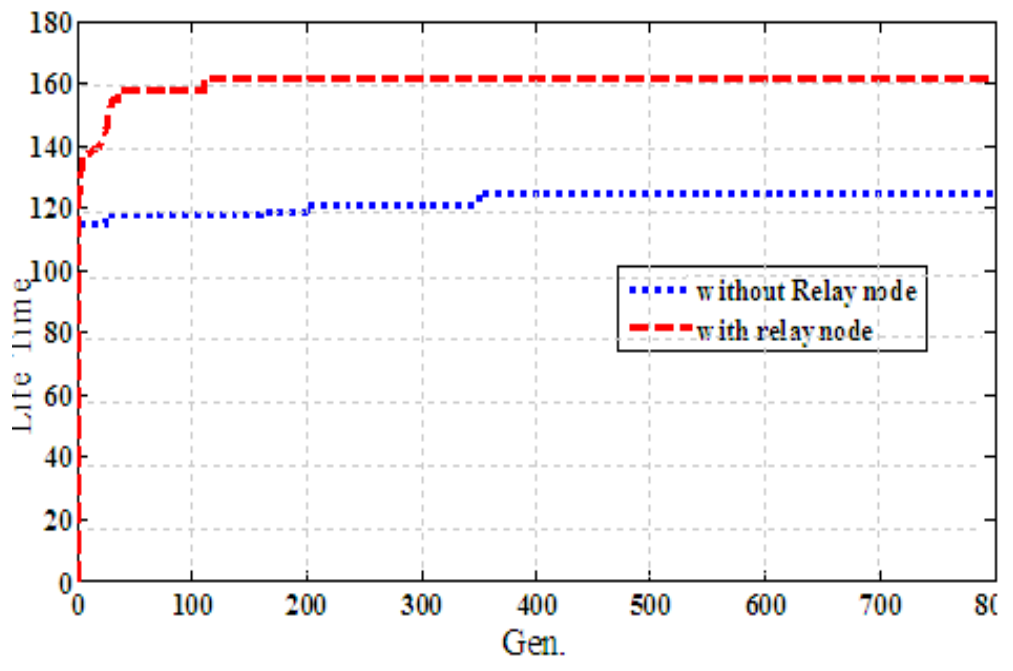

Figure 2. Lifetime Comparison

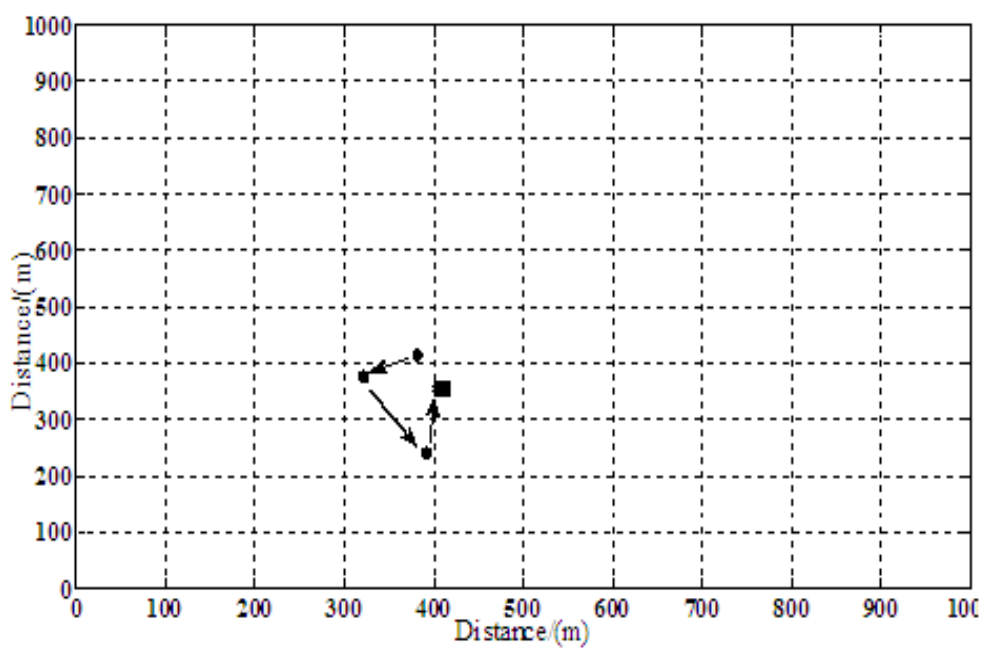

Figure 3. Optimal Sink Location (area = $1000 \mathrm{~m} \times 1000 \mathrm{~m}$ ) 


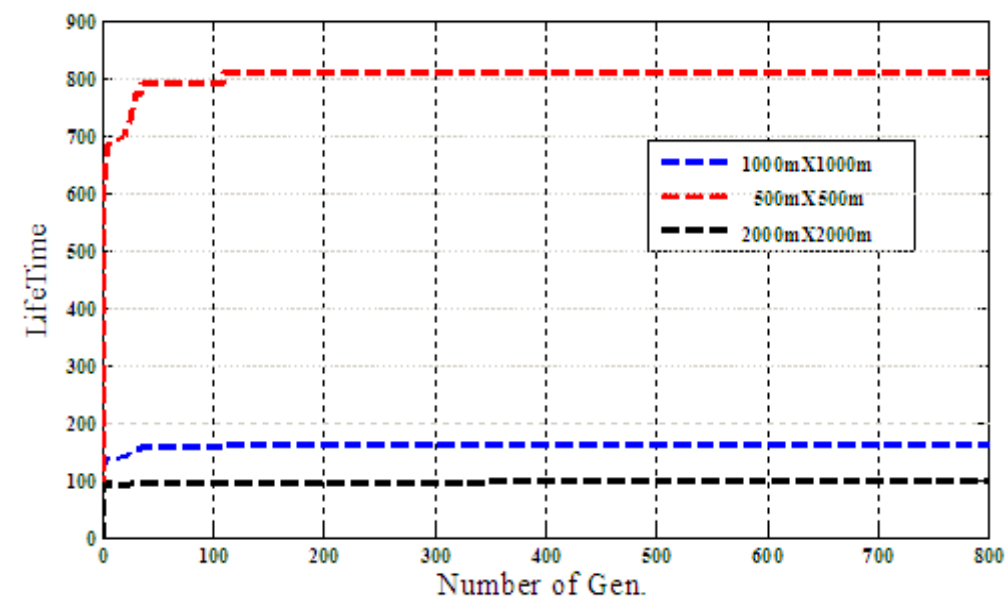

Figure 4. Lifetime Comparison with Respect To the Size of the Area

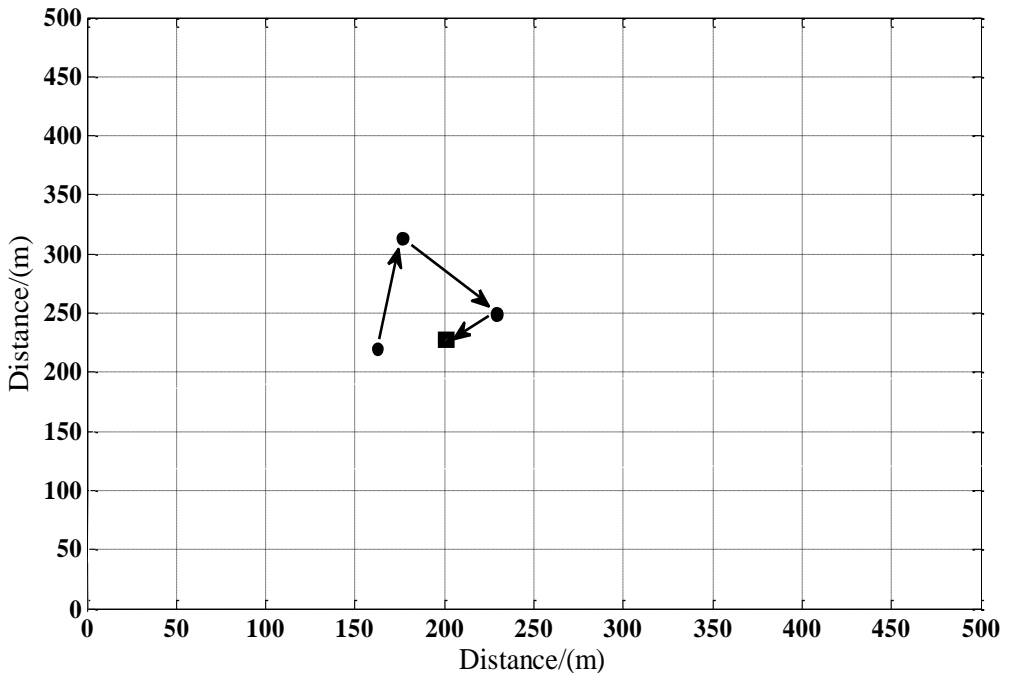

Figure 5. Optimal Sink Location (area $=500 \mathrm{~m} \times 500 \mathrm{~m}$ )

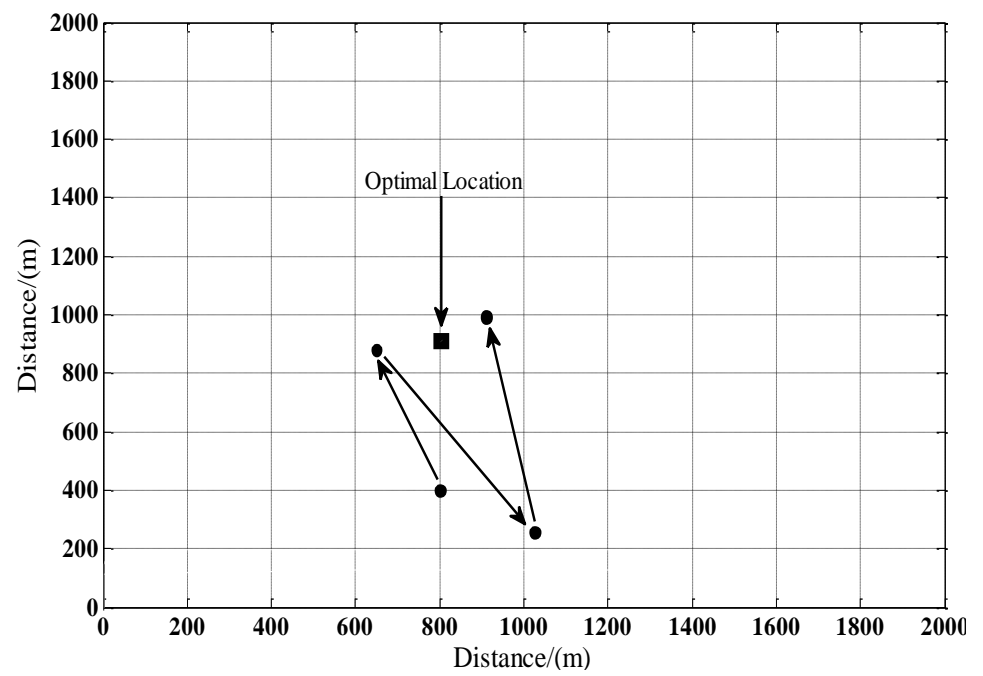

Figure 6. Optimal Sink Location (area $=2000 \mathrm{~m} \times \mathbf{2 0 0 0 \mathrm { m }}$ ) 


\section{Conclusion}

This paper presents a simple scheme for improving the network lifetime without compromising any of the severe requirements of wireless sensor networks. By utilizing relay nodes, the network is able to work for a longer lifetime. In order to utilize renewable-energy resources efficiently and economically, one optimum sizing method is developed in this paper based on a Biogeography-Based Optimization (BBO), which has the ability to attain the global optimum with relative computational simplicity compared to the conventional optimization methods.

The BBO algorithm optimizes the size and the operation strategy for a simple daily load. A scheme is proposed where relay nodes are used to collect data from the sensor nodes to pass them to the sink. We have calculated the required number of relay nodes to maintain the network connectivity successfully and then investigated their effect on network lifetime. The optimal location of the sink is determined with respect to those relay nodes with the help of the biogeography-based optimization technique. Experimental results show that the combination of the optimal location of the sink and relay nodes improves the network lifetime significantly. The $\mathrm{BBO}$ algorithm is able to optimize small as well as large lifetime systems. Therefore, this scheme can be used for designing a wireless sensor network with a longer lifetime.

\section{References}

[1] Y. Xu, J. Fang and W. Zhu, "Differential Evolution for Lifetime Maximization of Heterogeneous Wireless Sensor Networks," Mathematical Problems in Engineering, vol. 13, ID 172783, (2013).

[2] T. He, J. Stankovic and C. Lu, "A spatiotemporal communication protocol for wireless sensor networks," IEEE Trans. on Parallel and Distributed Systems, vol. 16, no. 10, (2005), pp. 995-1006.

[3] A. Rafael, J. Antonio and G. Felipe, "On Maximizing the Lifetime of Wireless Sensor Networks by Optimally Assigning Energy Supplies," Sensors, vol. 13, (2013), pp. 10219-10244.

[4] J. Tsitsiklis, "Decentralized detection," in Advances in Statistical Signal Processing, Greenwich, CT: JAI, vol. 2, (1993), pp. 297-344.

[5] R. Tenney and N.Sandell, "Detection with distributed sensors," IEEE Trans. Aerosp, Electron. Syst., vol. AES-17, no. 4, (1981) July, pp. 501-510.

[6] D. Simon, "Biogeography-based optimization," IEEE Trans. Evolutionary Computation, vol. 12, no. 6, (2008) December, pp. 702-713.

[7] Y. Liang, J. Cao and L. Zhang, "A biologically inspired sensor wakeup control method for wireless sensor networks," IEEE Trans. on Systems, Man and Cybernetics Part C., vol. 40, no. 5, (2010), pp. $525-538$.

[8] R. Tharmarasa, T. Kirubarajan and M. Hernandez, "Large scale optimal sensor array management for multi-target tracking," IEEE Trans. Systems, Man, and Cybernetics, Part C., vol. 37, no. 5, (2007), pp. 803-814.

[9] R. Tharmarasa, T. Kirubarajan and J. Peng, "Optimization-based dynamic sensor management for distributed multi-target tracking," IEEE Trans. on Systems, Man and Cybernetics Part C., vol. 39, no. 5, (2009), pp. 534-546.

[10] C. Chang, J. Sheu and Y. Chen, "An obstacle-free and power-efficient deployment algorithm for wireless sensor networks," IEEE Trans. on Systems, Man, and Cybernetics Part A., vol. 39, no. 4, (2009), pp. 795-806.

[11] F. Marcelloni and M. Vecchio, "A simple algorithm for data compression in wireless sensor networks," IEEE Communications Letters, vol. 12, no. 6, (2008), pp. 411-413.

[12] S. Yang, H. Cheng and F. Wang, "Genetic algorithms with immigrants and memory schemes for dynamic shortest path routing problems in mobile ad hoc networks," IEEE Trans. on Systems, Man and Cybernetics Part C., vol. 40, no. 1, (2010), pp. 52-63.

[13] S. Okdem and D. Karaboga, "Routing in wireless sensor networks using an Ant Colony optimization (ACO) router chip," Sensors, vol. 9, no. 2,(2009), pp. 909-921.

[14] F. Ge, Y. Wang and Q. Wang, "Energy efficient broadcasting based on ant colony optimization in wireless sensor networks," in Proceedings of the 3rd International Conference on Natural Computation ( ICNC '07), (2007), pp. 129-133.

[15] A. Acharya, A. Seetharam and A. Bhattacharyya, "Balancing energy dissipation in data gathering wireless sensor networks using ant colony optimization," in Proceedings of the International Conference on Distributed Computing and Networking (ICDCN '09), (2009), pp. 437-443. 
[16] H. Long, Y. Liu and Y. Wang, "Battery Allocation for Wireless Sensor Network Lifetime Maximization under Cost Constraints," in Proceedings of the International Conference on Computer-Aid Design, San Jose, CA, USA, (2009) November, pp. 2-5.

[17] G. Reise, J. Matamoros and C. Anton-Haro, "MSE-Optimal Power Allocation in Wireless Sensor Networks for Field Reconstruction Based on Shift-Invariant Spaces," in Proceedings of the Conference Record of the Forty Fifth Asilomar Conference on Signals, Systems and Computers, Pacific Grove, CA, USA, (2011) November, pp. 702-707.

[18] Y. Lin, J. Zhang and S. Chung, "An ant colony optimization approach for maximizing the lifetime of heterogeneous wireless sensor networks," IEEE Trans. on Systems, Man, and Cybernetics Part C., vol. 42, no. 3, (2012), pp. 408-420.

[19] M. Cardei and D. Zhang, "Improving wireless sensor network lifetime through power aware organization," Wireless Networks, vol. 11, no. 3, (2005), pp. 333-340.

[20] C. Lai, C. Ting and R. Ko, "An effective genetic algorithm to improve wireless sensor network lifetime for large scale

Surveillance applications," in Proceedings of the IEEE Congress on Evolutionary Computation (CEC '07), (2007), pp. 3531- 3538.

[21] X. Hu, J. Zhang and Y. Yu, "Hybrid genetic algorithm using a forward encoding scheme for lifetime maximization of wireless sensor networks," IEEE Trans. on Evolutionary Computation, vol. 14, no. 5, (2010), pp. 766-781.

[22] R. Storn and K. Price, "Differential evolution - a simple and efficient heuristic for global optimization over continuous spaces," Journal of Global Optimization, vol. 11, no. 4, pp. 341-359.

[23] W. Zhu, Y. Tangand, J. Fang, "Adaptive population tuning scheme for differential evolution," Information Sciences, vol. 223, (2013), pp. 164-19.

[24] M. Gidlund, "Performance of combined constellation rearrangement and space-time block coding scheme for multi-level modulation," in Proc. 18th IEEE PIMRC, Athens, Greece, (2007) September, pp. $1-5$.

[25] M. Ettus, "System capacity, latency, and power consumption in multi-hop routed SS-CDMA wireless networks," in Proc. Radio and Wireless Conf. Colorado Springs, USA, (1998), pp.55-58.

[26] Y. Hou, Thomas and Y. Shi, "Optimal base-station selection for any cast routing in wireless sensor networks," IEEE Trans. on Vehicular Technology, vol. 55, no. 3, (2006), pp.813-821. 